\title{
Intermittent theta burst stimulation enhances upper limb motor function in patients with chronic stroke: a pilot randomized controlled trial
}

Yu-Jen Chen ${ }^{1}$, Ying-Zu Huang ${ }^{2,3,4}$, Chung-Yao Chen ${ }^{4,5}$, Chia-Ling Chen ${ }^{1,6^{*}}$, Hsieh-Ching Chen ${ }^{7}$, Ching-Yi Wu ${ }^{1,8}$, Keh-Chung Lin ${ }^{9,10}$ and Tzu-ling Chang ${ }^{1}$

\begin{abstract}
Background: Intermittent theta burst stimulation (iTBS) is a form of repetitive transcranial stimulation that has been used to enhance upper limb (UL) motor recovery. However, only limited studies have examined its efficacy in patients with chronic stroke and therefore it remains controversial.

Methods: This was a randomized controlled trial that enrolled patients from a rehabilitation department. Twentytwo patients with first-ever chronic and unilateral cerebral stroke, aged 30-70 years, were randomly assigned to the iTBS or control group. All patients received 1 session per day for 10 days of either iTBS or sham stimulation over the ipsilesional primary motor cortex in addition to conventional neurorehabilitation. Outcome measures were assessed before and immediately after the intervention period: Modified Ashworth Scale (MAS), Fugl-Meyer Assessment Upper Extremity (FMA-UE), Action Research Arm Test (ARAT), Box and Block test (BBT), and Motor Activity Log (MAL). Analysis of covariance was adopted to compare the treatment effects between groups.

Results: The iTBS group had greater improvement in the MAS and FMA than the control group $\left(\eta^{2}=0.151-0.233\right.$; $p<0.05)$, as well as in the ARAT and BBT $\left(\eta^{2}=0.161-0.460 ; p<0.05\right)$ with large effect size. Both groups showed an improvement in the BBT, and there were no significant between-group differences in MAL changes.

Conclusions: The ITBS induced greater gains in spasticity decrease and UL function improvement, especially in fine motor function, than sham TBS. This is a promising finding because patients with chronic stroke have a relatively low potential for fine motor function recovery. Overall, iTBS may be a beneficial adjunct therapy to neurorehabilitation for enhancing UL function. Further larger-scale study is warranted to confirm the findings and its long-term effect.
\end{abstract}

Trial registration: This trial was registered under ClinicalTrials.gov ID No. NCT01947413 on September 20, 2013.

Keywords: Transcranial magnetic stimulation (TMS), Theta burst stimulation (TBS), Stroke, Motor function, Rehabilitation

\section{Background}

Stroke is the leading cause of long-term disability globally [1]. It is estimated that $55-75 \%$ of post-stroke patients experience upper limb (UL) functional limitation [2]. This UL functional impairment results in

\footnotetext{
* Correspondence: clingchen@gmail.com

'Department of Physical Medicine and Rehabilitation, Chang Gung Memorial Hospital, Linkou, 5, Fushing Street, Kuei-Shan District, Taoyuan City 33305, Taiwan

${ }^{6}$ Graduate Institute of Early Intervention, Chang Gung University, Taoyuan, Taiwan

Full list of author information is available at the end of the article
}

restrictions on functional tasks and daily activities [3], and may, therefore, lead to decreased health-related quality of life [4]. A reported $50-60 \%$ of patients experience variable degrees of motor function limitation following stroke, even when undergoing traditional rehabilitation programs [5]. Repetitive transcranial magnetic stimulation (rTMS) is a promising emerging non-invasive brain stimulation technique for facilitating functional recovery by modulating neuroplastic processes, together with post-stroke motor network activity and connectivity [6]. However, one meta-analysis

(C) The Author(s). 2019 Open Access This article is distributed under the terms of the Creative Commons Attribution 4.0 International License (http://creativecommons.org/licenses/by/4.0/), which permits unrestricted use, distribution, and 
[7] stated that the current literature is not sufficient to show the superiority of rTMS combined with UL training over UL training alone. Intermittent TBS (iTBS), however, may hold more promise; thus, we investigated its efficacy in the neurorehabilitation of UL dysfunction.

Theta burst stimulation (TBS) is a novel form of rTMS composed of short and low-intensity bursts of rTMS at $50 \mathrm{~Hz}$ [8]. Compared to conventional rTMS protocols, TBS yields consistent and long-lasting effects on motor-evoked potentials (MEPs) after a shorter stimulation duration [8]. In general, iTBS intermittently produces short TBS trains to facilitate cortical excitability of primary motor cortex (M1), while continuous TBS (cTBS) represents a longer and continuous TBS train that suppresses M1 cortical excitability. Notably, loci other than M1 may not induce similar effects $[9,10]$. Recent studies have shown controversial effects of TBS on UL function in patients with chronic stroke [11-16]. Based on the interhemispheric competition model, transcallosal inhibitory signals from the contralesional hemisphere hamper recovery of motor function post stroke [17], while cortical hyperexcitability in the contralesional hemisphere decreases with time after stroke [18] and may thus limit the effect of cTBS during the chronic stage. On the contrary, motor recovery has been associated with activation of the ipsilesional motor cortex in the chronic stroke stage [19], thus supporting the stimulation strategy of facilitating the ipsilesional primary motor cortex (M1). Therefore, iTBS was selected in this study to comprehensively elucidate its efficacy for the treatment of UL dysfunction in patients with chronic stroke.

The results of sham-controlled studies on the effect of iTBS on UL motor function in patients with chronic stroke have been controversial [13-16]. Three studies have shown that iTBS resulted in significant motor improvements $[13,15,16]$, while one study revealed no difference between the iTBS and sham groups [14]. One recent meta-analysis concluded that iTBS is more effective than CTBS for stroke-related UL motor deficits [20]. However, both patients with subacute and chronic stroke were included, and Egger's test revealed a significant publication bias [20]. To our knowledge, no previous studies have used spasticity as an outcome measure to evaluate the efficacy of iTBS combined with UL training. Furthermore, none have separately evaluated fine motor and gross motor functions of ULs. Therefore, as well as the common assessments of UL function, we also explored the effect of iTBS on these outcomes.

Motor recovery, especially fine motor, is usually limited beyond 6 months after stroke [1]. We tried to elucidate whether iTBS enhances fine motor function recovery in patients with chronic stroke. Based on previous randomized controlled trials (RCTs) of neurorehabilitation, we implemented a 2-week intervention [21, 22]. Outcome measures included the assessments of body function and activities based on the International Classification of Functioning, Disability, and Health (ICF) framework; the ICF can be a reference to determine and quantify the notion incorporated in outcome measures in stroke trials [23]. Body function was assessed using the modified Ashworth scale (MAS) [24] and Fugl-Meyer Assessment Upper Extremity (FMA-UE) [25], and activity measures used were the Action Research Arm Test (ARAT) [26], Box and Block test (BBT) [27], and motor activity log (MAL) [28]. The ARAT assesses both proximal and distal motor function and can, therefore, be used to examine fine and gross motor function separately. We hypothesized that 2-week iTBS combined with standard rehabilitation programs would induce greater improvements in the FMA and in spasticity in patients with chronic stroke compared to a sham intervention. We also expected that iTBS would result in greater improvements in the ARAT, BBT, and MAL. To our knowledge, this is the first RCT that compared the functional improvements in body function and activity domains, including spasticity, fine/gross motor function, and activities of daily living (ADL), post-stroke in patients undergoing real vs. sham iTBS.

\section{Methods \\ Participants}

Patients with stroke were enrolled from a rehabilitation department between 2013 and 2016. The diagnosis was confirmed by the authors according to clinical manifestations and imaging studies. Inclusion criteria for patients were as follows: (1) aged between 30 and 70 years; (2) first-ever ischemic or haemorrhagic stroke; (3) onset $\geq 6$ months; (4) unilateral cerebral stroke with hemiplegia or hemiparesis. Exclusion criteria were as follows: (1) brainstem or cerebellar stroke; (2) active psychiatric diseases; (3) severe psychological impairments, such as mental retardation, autism, or severe communication problems; (4) cognitive impairments that may interfere with understanding instructions; (5) progressive disorders, such as neurodegenerative disease; (6) an active medical condition, such as infection; (7) a history of seizure or aneurysm; (8) metal head implants.

All patients provided written informed consent. The study protocol was performed in accordance with the Declaration of Helsinki and was approved by a local institutional review board. This trial is registered under ClinicalTrials.gov ID No. NCT01947413.

\section{Design and procedures}

This study was a prospective, randomized, sham-controlled trial. Patients were blind to whether they received iTBS or sham-iTBS five times/week for 2 consecutive 
weeks. The flow diagram of the randomization procedure and the study design are illustrated in Figs. 1 and 2, respectively. The raters (occupational therapists who were only in contact with the patients during assessment), blinded to group assignment, were trained to administer outcome measures by senior therapists prior to the experiment, and passed a written competency and reliability test. Ten patients were selected for the intra-rater and inter-rater reliability tests at a 7-day interval. The intraclass correlations for intra-rater/inter-rater reliability of the MAS-UE, FMA-UE, ARAT, and BBT were 0.841/0.841, 0.984/0.992, $0.986 / 0.998$, and $1.000 / 0.998$, respectively.

The outcome measures were administered within 3 days before and after completing the 2-week iTBS/sham iTBS. All patients continued to participate in the standard in-patient rehabilitation programs, including conventional physical and occupational therapy $(90 \mathrm{~min} /$ session, 5 sessions/week), on the same day as the interventions. Demographic and clinical characteristics, such as age, sex, affected limb, stroke type, and lesion side, were recorded (Table 1).

\section{iTBS protocol}

iTBS was applied to the hand motor area of the affected hemisphere using a handheld figure-of-eight coil (70-mm standard coil; Magstim, Whitland, Dyfed, UK) connected to a Magstim Rapid ${ }^{2}$ stimulator. The optimal location of the coil was determined by the location on the scalp where the magnetic stimulation produced the largest MEP from the contralateral first dorsal interosseous muscle. True stimulation at $80 \%$ active motor threshold (AMT) targeted the identified hotspot, with the coil held tangentially to the skull, orientated at $45^{\circ}$ to the midsagittal axis, inducing posterior-anterior current in the brain. AMT was assessed before each intervention and was defined as the lowest transcranial magnetic stimulation (TMS) intensity to elicit MEPs of a $200 \mu \mathrm{V}$ peak-to-peak amplitude in 5 of 10 successive trials during slight contraction (10-20\% of maximum) of the target muscle. Sham stimulation was administered on the same site with the coil flipped over at a lower intensity (60\% AMT), which results in a lower output equivalent to that of the normal site at approximately

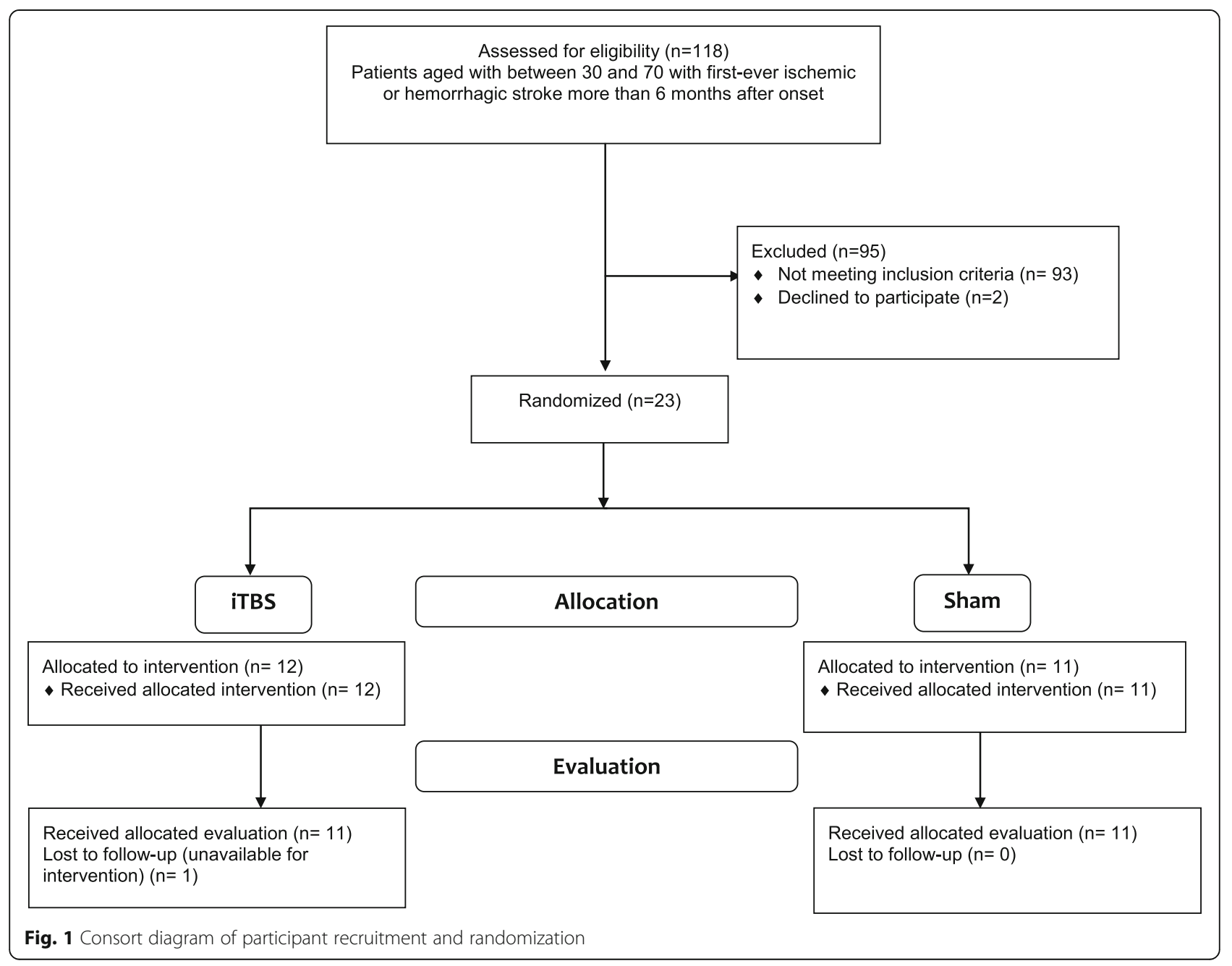




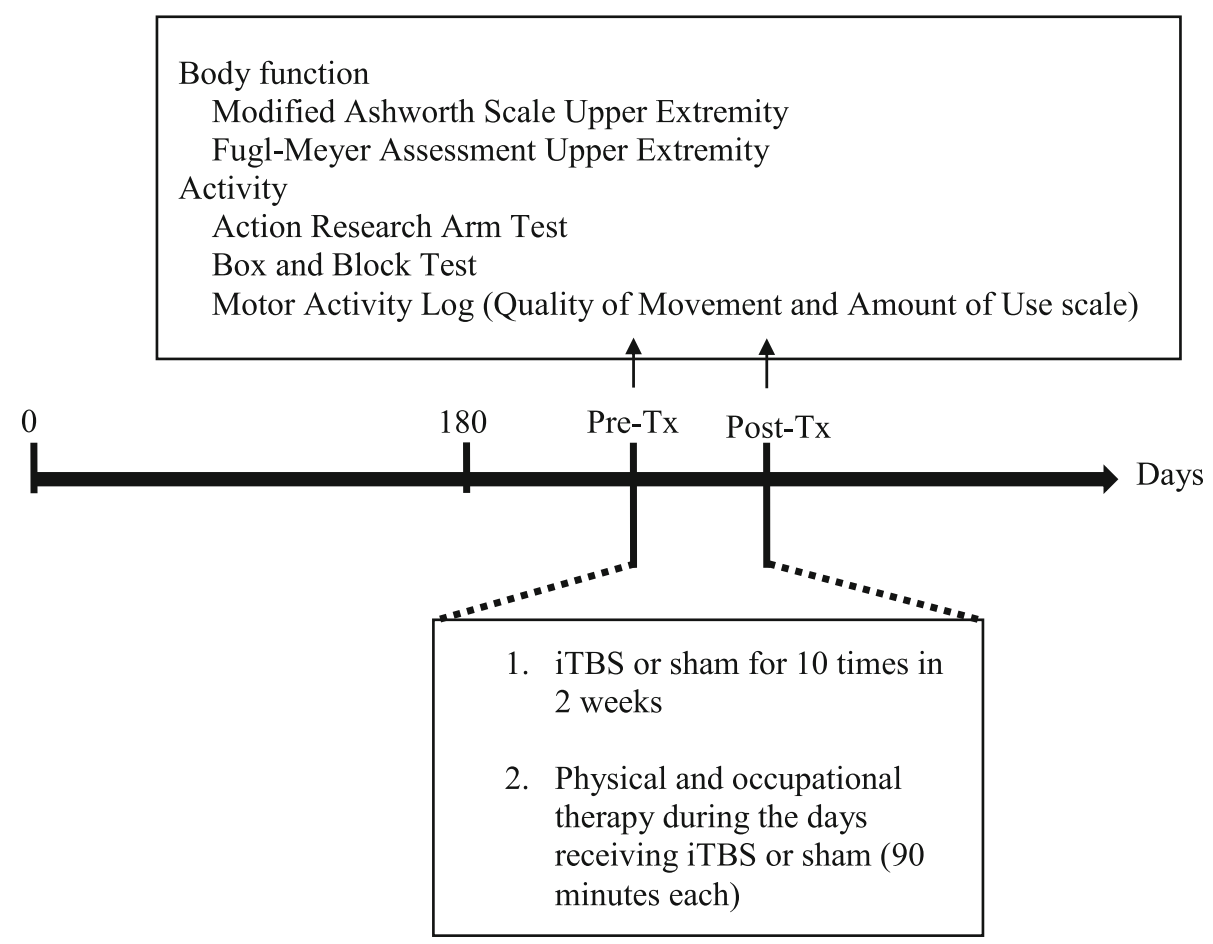

Tx Treatment

Fig. 2 Study design

Table 1 Demographic and clinical data

\begin{tabular}{llll}
\hline Variables & iTBS $(n=11)$ & Control $(n=11)$ & $p$ value \\
\hline Age (years) & $52.9 \pm 11.1$ & $52.6 \pm 8.3$ & $0.937^{\mathrm{a}}$ \\
Gender & $7 / 4$ & $7 / 4$ & $>0.99^{\mathrm{b}}$
\end{tabular}

Clinical characteristics

$\begin{array}{llll}\begin{array}{l}\text { Stroke type } \\ \text { Hemorrhage }\end{array} & 9(82 \%) & 8(73 \%) & >0.99^{\mathrm{b}} \\ \text { Infarction } & 2(18 \%) & 3(27 \%) & \\ \begin{array}{l}\text { Stroke side } \\ \text { Right }\end{array} & 5(45 \%) & 2(18 \%) & 0.170^{\mathrm{b}} \\ \text { Left } & 6(55 \%) & 9(82 \%) & \\ \text { Stroke location } & & & \\ \text { Supratentorial } & 4(36 \%) & 3(27 \%) & 0.647^{\mathrm{b}} \\ \text { Infratentorial } & 7(64 \%) & 8(73 \%) & \\ \text { MMSE } & 26.9 \pm 3.4 & 23.8 \pm 7.3 & 0.220^{\mathrm{a}} \\ \text { NIHSS } & 3.7 \pm 2.5 & 5.9 \pm 5.6 & 0.251^{\mathrm{a}}\end{array}$

Medications

$\begin{array}{llll}\text { Antispasticity } & 2(18 \%) & 3(27 \%) & 0.611^{\mathrm{b}}\end{array}$

Data are expressed as mean \pm SD or $\mathrm{N}(\%)$

${ }^{\mathrm{a}} \mathrm{t}$ tests; ${ }^{\mathrm{b}}$ Chi-square tests

iTBS intermittent theta burst stimulation, MMSE Mini-mental state

examination, NIHSS National Institutes of Health Stroke Scale
46.8\% AMT, because the output of the flip side is about $78 \%$ of the normal side [29]. Previous study demonstrated that a single theta burst with intensity lower than 70\% AMT has no effect on MEPs [30]. The sound and sensation were similar to real stimulation so that the patients cannot differentiate. Similar sham stimulation method has been applied in several previous studies and proved to be useful $[29,31]$. When MEPs could not be measured from the affected side, iTBS was applied to the mirror location of the hotspot of the sound hemisphere (non-lesional M1) at the highest intensity that our $\mathrm{Rapid}^{2}$ could generate for TBS $(51 \%$ of maximum stimulator output (MSO)). Patients who had no measurable MEP on the affected side and who required stimulus intensity ( $80 \%$ AMT) higher than 51\% of MSO were also stimulated at $51 \%$ of MSO. No patient was able to distinguish whether they had received real or sham stimulation. Patients were instructed to relax their hands at all times during the experiment once the AMT had been recorded.

Real or sham iTBS was delivered at the same time of day for 5 consecutive days per week for 2 weeks. iTBS gave a 2-s train of bursts, which contained three $50-\mathrm{Hz}$ pulses repeated every $200 \mathrm{~ms}$ (i.e., $5 \mathrm{~Hz}$ ) at an intensity of $80 \%$ AMT, every $10 \mathrm{~s}$ for 20 times (600 pulses in total) [8]. Similar stimulation parameters have been applied in several previous studies $[21,32]$. 


\section{Outcome measures MAS}

MAS [24] was used for determining muscle spasticity. We summed the MAS scores of the affected elbow, wrist, and finger flexor muscles to evaluate UL spasticity. $1+$ was calculated as 1.5 for summation.

\section{FMA-UE}

The FMA [25] is a performance-based quantitative evaluation specifically used for measuring post-stroke impairments, including motor function, balance, sensation, and joint functions, and UL motor function evaluation was selected in the current study.

\section{ARAT}

The ARAT [26] is a measure of UL motor function after stroke which comprises of 4 subsections: grasp, grip, pinch, and gross movement.

\section{$B B T$}

The BBT [27] assesses grasping, transporting, and releasing of small objects to determine unilateral gross manual dexterity.

\section{MAL}

The MAL [28] is a structured interview that measures the use of the impaired arm in patients with stroke, which is used to obtain information about 14 activities of daily living.

\section{Statistical analysis}

Data analyses were conducted by H.L. Peng who was blinded to group allocation. To determine the baseline comparability of demographic characteristics, chi-square tests and independent two-sample t-tests were applied to categorical and continuous variables, respectively. Analysis of covariance was used to test whether the iTBS group showed greater improvement than the control group after treatment. We defined pre-treatment performance as the covariate, group as the independent variable, and post-treatment performance as the dependent variable. Effect size $\left(\eta^{2}\right)$ was calculated for each outcome variable to indicate the degree of group differences. A large effect is represented by an $\eta^{2}$ of at least 0.138 , a moderate effect by an $\eta^{2}$ of 0.059 , and a small effect by an $\eta^{2}$ of 0.01 [33]. Significance was set at 0.05 (one-tailed).

\section{Results}

A total of 118 patients were screened. Twenty-three were eligible for inclusion and were randomly assigned to the iTBS or control group by a research assistant using a computer random number generator. Of these 23 , one patient in the iTBS group was lost to follow-up due to unavailability for intervention. Finally, 11 patients in the iTBS group and 11 in the sham-iTBS group completed the intervention and evaluation. There were no adverse events throughout the study course. There was no significant between-group difference before treatment on demographic characteristics (Table 1) or outcome measures (Table 2). AMT cannot be measured in 4 patients in the iTBS group and 5 patients in the control group. The mean \pm standard deviation from the obtained AMT data of iTBS and control groups were $67 \% \pm 11$ and $71 \% \pm 8 \%$, respectively.

The iTBS group had greater gains in the MAS and FMA, with larger effects than the control group after the intervention (MAS: $\mathrm{F}_{1,19}=5.8, \quad p=0.014, \quad \eta^{2}=0.233$; FMA-UE: $\mathrm{F}_{1,19=} 3.4, p=0.046, \eta^{2}=0.151$ ) (Table 2, Fig. 3). Moreover, the iTBS group had greater gains in the ARAT in various domains, with larger effect sizes than the control group (average: $\mathrm{F}_{1,19}=16.3, p=0.001$, $\eta^{2}=0.460$; grasp: $\mathrm{F}_{1,19}=7.6, p=0.006, \eta^{2}=0.286$; grip: $\mathrm{F}_{1,19}=3.7, p=0.035, \eta^{2}=0.163$; pinch: $\mathrm{F}_{1,19}=11.9, p=$ $\left.0.002, \eta^{2}=0.384\right)$.

After treatment, the iTBS group had significantly greater gains in the BBT with a large effect size than the control group $\left(\mathrm{F}_{1,19}=3.7, p=0.036, \eta^{2}=0.161\right)$, although the BBT score of both groups improved. There were no significant between-group differences in the changes in the MAL Amount of Use (AOU) scale and MAL Quality of Movement (QOM) scale.

\section{Discussion}

In this pilot study, iTBS induced greater gains in the MAS and FMA than sham stimulation, and more greatly improved BBT and ARAT scores, especially for fine motor function. However, the changes in the MAL did not differ between the two groups. These findings suggest that iTBS combined with conventional neurorehabilitation reduces spasticity and improves UL motor function, but not ADL, in patients with chronic stroke. The beneficial effect of iTBS in patients with chronic stroke is even more striking if we consider that the likelihood of fine motor recovery is lower in chronic phases than in the subacute or acute stroke. Overall, although limited by the relatively small sample size, these findings suggest that iTBS may be used as an adjunct therapy to enhance UL function in patients with chronic stroke.

\section{The effect of iTBS on upper limb motor function}

The application of iTBS to the ipsilesional hemisphere enhanced UL motor function, as measured by the FMA, ARAT, and BBT. The iTBS-induced changes in these outcome measures achieved large effect sizes, although the changes in FMA and ARAT were not greater than the minimal clinically important difference [34, 35]. These results are consistent with those of previous 
Table 2 Descriptive and inferential statistics for analysis of outcome measures

\begin{tabular}{|c|c|c|c|c|c|c|c|c|}
\hline \multirow[t]{2}{*}{ Variables } & \multicolumn{3}{|l|}{ Pre-treatment } & \multicolumn{2}{|l|}{ Post-treatment } & \multicolumn{3}{|c|}{ ANCOVA } \\
\hline & $\mathrm{iTBS}(n=11)$ & Control $(n=11)$ & $p$ value ( $t$ test) & $\mathrm{iTBS}(n=11)$ & Control $(n=11)$ & $F 1,19$ & $p$ value & Effect size $\eta 2$ \\
\hline MAS-UE & $3.90 \pm 2.10$ & $4.05 \pm 1.56$ & 0.861 & $3.30 \pm 1.98$ & $4.29 \pm 1.44$ & 5.8 & $0.014^{*}$ & 0.233 \\
\hline FMA-UE & $33.33 \pm 19.80$ & $30.03 \pm 22.11$ & 0.732 & $34.65 \pm 19.80$ & $27.06 \pm 20.79$ & 3.4 & $0.046^{*}$ & 0.151 \\
\hline ARAT & $18.62 \pm 19.38$ & $16.72 \pm 22.8$ & 0.834 & $23.18 \pm 20.71$ & $13.3 \pm 19.95$ & 16.3 & $0.001+$ & 0.460 \\
\hline Gross movement & $9.06 \pm 7.08$ & $7.98 \pm 6.48$ & 0.708 & $10.02 \pm 6.60$ & $6.72 \pm 6.18$ & 2.1 & 0.083 & 0.099 \\
\hline Grasp & $4.00 \pm 4.48$ & $3.52 \pm 5.28$ & 0.819 & $4.60 \pm 4.96$ & $2.68 \pm 4.72$ & 7.6 & $0.006+$ & 0.286 \\
\hline Grip & $4.80 \pm 7.08$ & $5.58 \pm 7.86$ & 0.800 & $6.42 \pm 7.20$ & $5.04 \pm 7.38$ & 3.7 & $0.035^{*}$ & 0.163 \\
\hline Pinch & $2.46 \pm 3.39$ & $1.86 \pm 3.51$ & 0.692 & $3.51 \pm 3.21$ & $1.26 \pm 2.82$ & 11.9 & $0.002 \dagger$ & 0.384 \\
\hline BBT & $10.20 \pm 16.80$ & $9.00 \pm 21.00$ & 0.920 & $11.40 \pm 18.60$ & $9.60 \pm 22.20$ & 3.7 & $0.036^{*}$ & 0.161 \\
\hline MAL (AOU) & $10.44 \pm 16.38$ & $18.9 \pm 22.96$ & 0.460 & $12.18 \pm 14.70$ & $20.72 \pm 22.54$ & 0.02 & 0.460 & 0.001 \\
\hline MAL (QOM) & $10.50 \pm 13.44$ & $15.26 \pm 22.40$ & 0.580 & $10.36 \pm 13.86$ & $21.28 \pm 22.12$ & 0.5 & 0.246 & 0.031 \\
\hline
\end{tabular}

Values are expressed as mean \pm SD

iTBS intermittent theta burst stimulation, MAS-UE Modified Ashworth Scale Upper Extremity, FMA-UE Fugl-Meyer Assessment Upper Extremity, ARAT Action Research Arm Test, BBT Box and Block test, MAL(AOU) Motor Activity Log (Amount of Use), MAL (QOM) Motor Activity Log (Quality of Movement) ${ }^{*} p<0.05 ;+p<0.01$

studies on iTBS [13-15] and conventional high-frequency facilitatory rTMS $[22,36]$ in stroke patients, although iTBS may not enhance UL motor function in patients with incomplete spinal cord injury [37]. Our results may be explained by vicariation [38, 39]. The vicariation theory postulates that brain areas are reorganized to substitute the functions of nearby injured areas
[38, 39]. Considering this compensatory neural plasticity, facilitatory stimulation should be applied to the affected hemisphere in order to enhance excitability of compensatory neurons [39]. According to this model, facilitation of the ipsilesional hemisphere may stimulate cerebral tissue reorganization and restore the balance of motor cortical excitability between

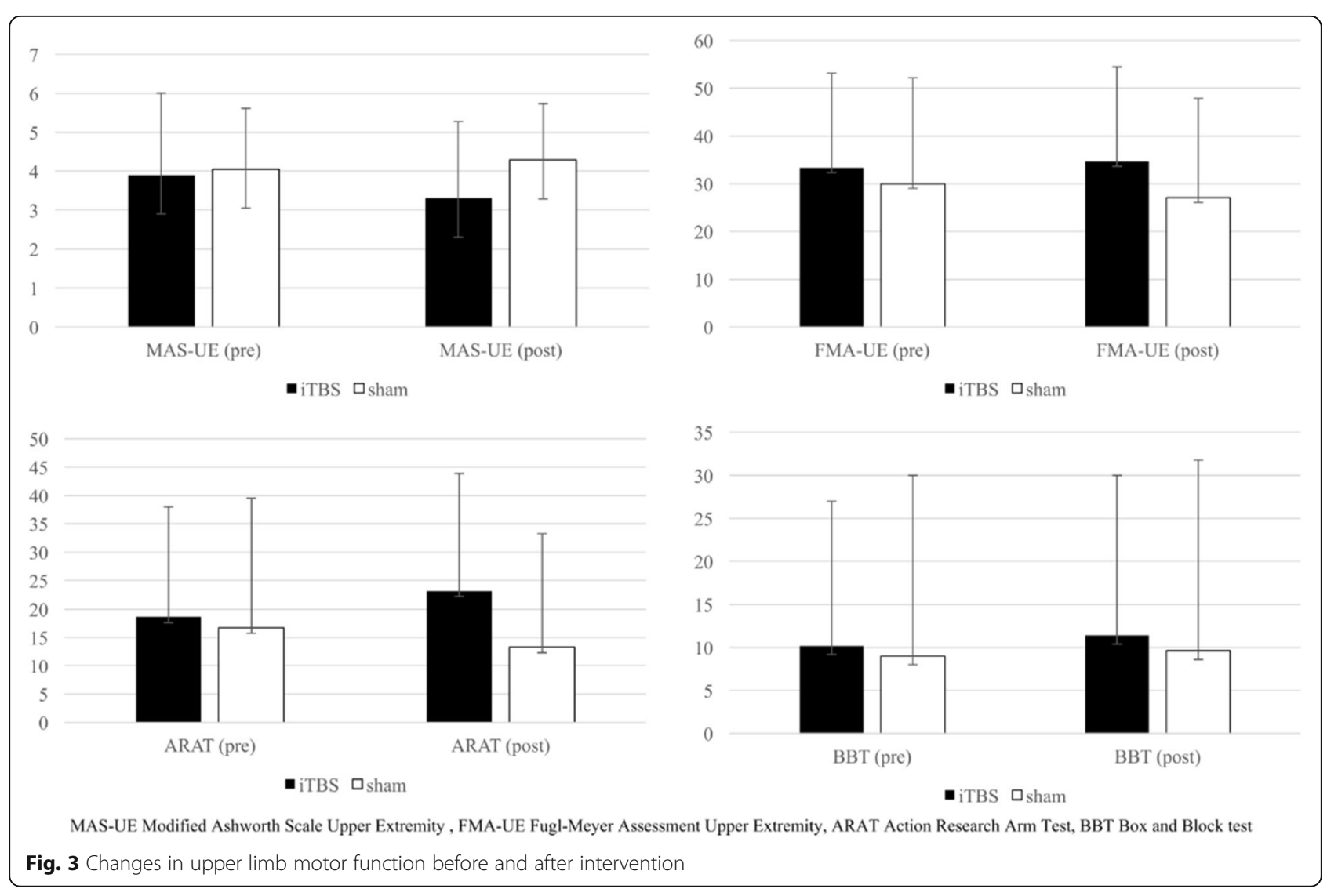


hemispheres, thus leading to functional recovery of the affected limb.

Our findings that the iTBS group had greater improvements than the control group, in pinch, grasp, and grip functions, but not in gross movement, suggest that iTBS can enhance fine UL movement to a greater degree than gross UL movement. Our results were partially compatible with those of previous iTBS studies [13-16]. For example, Talelli et al. first applied iTBS as an intervention to improve UL motor function recovery in 6 patients with chronic stroke, and found that simple reaction times of gripping a dynamometer were improved [13]. Ackerley et al. recruited 10 patients with subcortical CS and found that iTBS followed by motor training enhanced performance in grip-lift kinetics of the affected hand [15]. Another study by Ackerley et al. also revealed that iTBS induced significant improvements in the ARAT [16]. However, another study by Talelli et al. demonstrated that there were no significant differences in the grasp and pinch strength or performance in the Nine-Hole Peg Test and Jebson Taylor Test between iTBS and sham groups post-treatment and at all follow-ups (4, 30, and 90 days), although motor performance slightly improved in both groups [14]. It is generally accepted that the proximal limb motor (gross motor) function recovers better than the function of distal parts (fine motor) after stroke [40], possibly because the recovery of gross motor function can be mitigated by multiple neural pathways [41], while fine motor function is primarily controlled locally by the M1 and corticospinal tract [42]. We selected the first dorsal interosseous muscle for AMT determination, which mainly accounts for fine motor function, and this may account for our results. However, gross motor movement is dependent on shoulder and elbow function. Our findings suggest that iTBS enhances fine motor recovery, even in patients with chronic stroke.

This is the first study to verify the efficacy of iTBS combined with UL training in reducing spasticity in patients with chronic stroke. It has been postulated that impaired cortical motor neurons after stroke cannot sustain inhibitory signals to the corticospinal tract, leading to an increased spinal motor neuron excitability that results in spasticity [43]. Facilitatory rTMS, including iTBS, has been reported to reduce spasticity in patients with multiple sclerosis [44, 45], cerebral palsy [46], and spinal cord injury $[47,48]$. In patients with acute and chronic stroke, Kim et al. found that a single session of iTBS resulted in a transient reduction of spasticity [49]. In another study with patients with chronic stroke, facilitatory rTMS decreased the F-waves, implying that facilitatory rTMS suppresses the excitability of the spinal cord by enhancing inhibitory inputs from the cortex to spinal neurons [50]. In addition, iTBS may cause changes in endogenous neurotransmitters including $\gamma$-aminobutyric acid [51], glutamate [52], and dopamine [53], which are involved in synaptic plasticity, thereby increasing the efficacy of synaptic transmission [54]. As a result, it may facilitate inhibitory interneurons from the cortex to the spinal cord and reduce spasticity. Further trials with additional neurophysiological studies to examine the effects induced by iTBS on corticospinal tract excitability are warranted to uncover its underlying neuronal mechanisms and long-term clinical effects.

The iTBS group had greater gains in the BBT than the control group, which indicates that iTBS may enhance the recovery of gross manual dexterity of UL in patients with chronic stroke. Our results were not compatible with those of a previous iTBS study [14], but partially compatible with those of rTMS studies [55, 56]. Namely, Talelli et al. revealed no difference in the Nine Hole Peg Test after intervention between the iTBS and sham groups in patients with chronic stroke [14]. One meta-analysis showed no significant differences in the BBT between rTMS and conventional rehabilitation programs [7]. However, one RCT found that the facilitatory rTMS group had significantly greater improvements on BBT than a sham group, up until 6 months post-intervention [55]. Overall, these variable findings may be due to different patient characteristics, stimulation protocols, and/or stimulation locations among studies. Furthermore, the effects of rTMS on neural activity depend on factors such as coil orientation, stimulation intensity, and brain activation [57]. For example, the TMS-related effect has been linked to the total number of stimuli, whereby a profound change in cortical excitability was observed for longer periods of rTMS [58]. TBS and paired associative stimulation modulate different synapses in the motor cortex, although both modulate cortical excitability [59]. Our finding suggests that iTBS may be used to enhance the gross manual dexterity in patients with chronic stroke.

In the current study, MAS, FMA, and ARAT scores was decreased after intervention in the control group, which were also demonstrated in previous literature using a different sham stimulation method [16]. One possible explanation is that the facilitatory effect of iTBS outweigh the exhaustibility from rehabilitation, so the exhaustibility caused by extensive rehabilitation is only seen in the control group having no improvement.

The iTBS group did not show greater improvement in the MAL (QOM) or MAL (AOU) than the control group. This is consistent with previous facilitatory rTMS results [60]. Malcolm et al. failed to identify a superiority of 10 sessions of $20 \mathrm{~Hz}$ rTMS to the ipsilesional hemisphere over a sham intervention in terms of MAL (QOM) and MAL (AOU) at 2-week and 6-month follow-ups [55]. MAL has been used to capture changes 
in the actual use of the affected arm that differ from compensatory strategies post stroke, and is dissimilar to laboratory-based function measurements [61]. A possible explanation for this result is that the follow-up duration was too short to detect the ADL changes and learned non-use, thus reinforcing the use of compensatory strategies despite gradual functional recovery [62].

\section{Study limitations}

There were several limitations to this study. First, as with other iTBS studies [13-15, 21], the sample size was relatively small; the results should be confirmed in a larger-scale study. Second, the follow up duration was too short to assess the long-term impact of iTBS; future studies should also investigate the persistence of these effects. Third, real or sham iTBS was arranged at the available time based on the daily regime of neurorehabilitation. It is not clear whether the effect of iTBS is associated with the stimulation timing relative to neurorehabilitation or not. Fourth, high AMT was likely due to the severely damaged motor pathway. Such high motor thresholds led to insufficient stimulus intensity due to the limitation of the TMS machine, and could lower the efficacy of iTBS. Fifth, the sham stimulation method allows the intensity to be delivered at a decreased magnitude rather than abolishing it. In addition, the sensation on the scalp may be similar but could not be identical with that of the iTBS group.

\section{Conclusions}

iTBS over the affected hemisphere reduced spasticity and improved UL motor function, particularly fine motor function, which is not usually the case in the chronic stage of stroke. To conclude, iTBS is a non-invasive and safe neuromodulatory stimulation technique that may be a beneficial adjunct to neurorehabilitation for patients with chronic stroke who have relatively low potential for motor recovery. While the current study is a pilot study with small sample size, further larger-scale study is needed to confirm the findings.

\section{Abbreviations \\ ADL: Activities of daily living; AMT: Active motor threshold; AOU: Amount of Use scale; ARAT: Action Research Arm Test; BBT: Box and Block test; CTBS: Continuous TBS; FMA-UE: Fugl-Meyer Assessment Upper Extremity; ICF: International Classification of Functioning, Disability, and Health framework; iTBS: Intermittent TBS; M1: Primary motor cortex; MAL: Motor activity log; MAS: Modified Ashworth scale; MEPs: Motor-evoked potentials; QOM: Quality of Movement scale; RCT: Randomized controlled trial; rTMS: Repetitive transcranial magnetic stimulation; TBS: Theta burst stimulation; TMS: Transcranial magnetic stimulation; UL: Upper limb}

\section{Acknowledgements}

We thank the patients who participated in this study.

\section{Funding}

The National Science Council of Taiwan (MOST, 105-2314-B-182 -020 -MY3) and Chang Gung Memorial Hospital, Taiwan (CMRPG 3D1461-3, CMRPG
3B1441-3 and CMRPG 3F1251) partially/financially supported this research. The funding bodies had no role in the study design, data collection, data analysis, data interpretation and writing of the manuscript.

Availability of data and materials

The data used and/or analysed of the current study are available from the corresponding author on reasonable request.

\section{Authors' contributions}

CYJ acquired the data, analyzed and interpreted the data and was the author drafting the paper. CCL was involved in the conception and design of the study, in the analysis and interpretation of the results and in writing the paper. HYZ was involved in the design of the study, in the analysis and interpretation of the results and in writing the paper. CCY, CHC, WCY, LKC and $C Z L$ were involved in the design of the study, in the interpretation of the results and in writing the paper. All authors read and approved the final manuscript.

\section{Ethics approval and consent to participate}

The study protocol was performed in accordance with the Declaration of Helsinki and was approved by local ethics committee of Chang Gung Memorial Hospital, Taiwan. All patients gave written informed consent prior to participation.

\section{Consent for publication}

Not applicable.

\section{Competing interests}

The authors report no conflicts of interest. The authors alone are responsible for the content and writing of the paper. No party has direct interest neither a financial relationship nor will they be conferred any benefits from writing this submission or the results of this research.

\section{Publisher's Note}

Springer Nature remains neutral with regard to jurisdictional claims in published maps and institutional affiliations.

\section{Author details}

${ }^{1}$ Department of Physical Medicine and Rehabilitation, Chang Gung Memorial Hospital, Linkou, 5, Fushing Street, Kuei-Shan District, Taoyuan City 33305,

Taiwan. ${ }^{2}$ Neuroscience Research Center and Department of Neurology, Chang Gung Memorial Hospital, Linkou, Taiwan. ${ }^{3}$ Institute of Cognitive Neuroscience, National Central University, Taoyuan, Taiwan. ${ }^{4}$ Medical School, College of Medicine, Chang Gung University, Taoyuan, Taiwan. ${ }^{5}$ Department of Physical Medicine and Rehabilitation, Chang Gung Memorial Hospital, Keelung, Taiwan. ${ }^{6}$ Graduate Institute of Early Intervention, Chang Gung University, Taoyuan, Taiwan. ${ }^{7}$ Department of Industrial and Management, National Taipei University of Technology, Taipei, Taiwan. ${ }^{8}$ Department of Occupational Therapy, College of Medicine, Chang Gung University, Taoyuan, Taiwan. ${ }^{9}$ School of Occupational Therapy, College of Medicine, National Taiwan University, Taipei, Taiwan. ${ }^{10}$ Division of Occupational Therapy, Department of Physical Medicine and Rehabilitation, National Taiwan University Hospital, Taipei, Taiwan.

Received: 26 March 2018 Accepted: 11 April 2019

Published online: 25 April 2019

References

1. Feigin $\mathrm{VL}$, Forouzanfar MH, Krishnamurthi $\mathrm{R}$, Mensah GA, Connor M, Bennett DA, Moran AE, Sacco RL, Anderson L, Truelsen T, et al. Global and regional burden of stroke during 1990-2010: findings from the global burden of disease study 2010. Lancet (London, England). 2014;383(9913):245-54.

2. Wolf SL, Winstein CJ, Miller JP, Taub E, Uswatte G, Morris D, Giuliani C, Light KE, Nichols-Larsen D. Effect of constraint-induced movement therapy on upper extremity function 3 to 9 months after stroke: the EXCITE randomized clinical trial. Jama. 2006:296(17):2095-104.

3. Hankey GJ, Jamrozik K, Broadhurst RJ, Forbes S, Anderson CS. Long-term disability after first-ever stroke and related prognostic factors in the Perth community stroke study, 1989-1990. Stroke. 2002;33(4):1034-40. 
4. Nichols-Larsen DS, Clark PC, Zeringue A, Greenspan A, Blanton S. Factors influencing stroke survivors' quality of life during subacute recovery. Stroke. 2005;36(7):1480-4.

5. Hendricks HT, van Limbeek J, Geurts AC, Zwarts MJ. Motor recovery after stroke: a systematic review of the literature. Arch Phys Med Rehabil. 2002:83(11):1629-37.

6. Grefkes C, Fink GR. Disruption of motor network connectivity post-stroke and its noninvasive neuromodulation. Curr Opin Neurol. 2012;25(6):670-5.

7. Graef P, Dadalt ML, Rodrigues DA, Stein C, Pagnussat Ade S. Transcranial magnetic stimulation combined with upper-limb training for improving function after stroke: a systematic review and meta-analysis. J Neurol Sci. 2016;369:149-58.

8. Huang YZ, Edwards MJ, Rounis E, Bhatia KP, Rothwell JC. Theta burst stimulation of the human motor cortex. Neuron. 2005;45(2):201-6.

9. Tsang P, Jacobs MF, Lee KGH, Asmussen MJ, Zapallow CM, Nelson AJ. Continuous theta-burst stimulation over primary somatosensory cortex modulates short-latency afferent inhibition. Clin Neurophysiol. 2014;125(11): 2253-9

10. Jacobs MF, Tsang P, Lee KG, Asmussen MJ, Zapallow CM, Nelson AJ. $30 \mathrm{~Hz}$ theta-burst stimulation over primary somatosensory cortex modulates corticospinal output to the hand. Brain Stimul. 2014;7(2):269-74.

11. Yamada N, Kakuda W, Kondo T, Shimizu M, Sageshima M, Mitani S, Abo M. Continuous theta-burst stimulation combined with occupational therapy for upper limb hemiparesis after stroke: a preliminary study. Acta Neurol Belg. 2014;114(4):279-84.

12. Di Lazzaro V, Rothwell JC, Talelli P, Capone F, Ranieri F, Wallace AC, Musumeci G, Dileone M. Inhibitory theta burst stimulation of affected hemisphere in chronic stroke: a proof of principle, sham-controlled study. Neurosci Lett. 2013;553:148-52.

13. Talelli P, Greenwood RJ, Rothwell JC. Exploring Theta burst stimulation as an intervention to improve motor recovery in chronic stroke. Clin Neurophysiol. 2007;118(2):333-42.

14. Talelli P, Wallace A, Dileone M, Hoad D, Cheeran B, Oliver R, VandenBos M, Hammerbeck U, Barratt K, Gillini C, et al. Theta burst stimulation in the rehabilitation of the upper limb: a semirandomized, placebocontrolled trial in chronic stroke patients. Neurorehabil Neural Repair. 2012;26(8):976-87.

15. Ackerley SJ, Stinear CM, Barber PA, Byblow WD. Combining theta burst stimulation with training after subcortical stroke. Stroke. 2010;41(7):1568-72.

16. Ackerley SJ, Byblow WD, Barber PA, MacDonald H, Mclntyre-Robinson A, Stinear CM. Primed physical therapy enhances recovery of upper limb function in chronic stroke patients. Neurorehabil Neural Repair. 2016;30(4):339-48.

17. Nowak DA, Grefkes C, Ameli M, Fink GR. Interhemispheric competition after stroke: brain stimulation to enhance recovery of function of the affected hand. Neurorehabil Neural Repair. 2009;23(7):641-56.

18. Ward NS, Brown MM, Thompson AJ, Frackowiak RS. Neural correlates of motor recovery after stroke: a longitudinal fMRI study. Brain : a journal of neurology. 2003;126(Pt 11):2476-96.

19. Marshall RS, Perera GM, Lazar RM, Krakauer JW, Constantine RC, DeLaPaz RL. Evolution of cortical activation during recovery from corticospinal tract infarction. Stroke. 2000;31(3):656-61

20. Zhang L, Xing G, Fan Y, Guo Z, Chen H, Mu Q. Short- and long-term effects of repetitive Transcranial magnetic stimulation on upper limb motor function after stroke: a systematic review and meta-analysis. Clin Rehabil. 2017:31(9):1137-53.

21. Hsu YF, Huang YZ, Lin YY, Tang CW, Liao KK, Lee PL, Tsai YA, Cheng HL, Cheng $\mathrm{H}$, Chern $\mathrm{CM}$, et al. Intermittent theta burst stimulation over ipsilesional primary motor cortex of subacute ischemic stroke patients: a pilot study. Brain Stimul. 2013;6(2):166-74.

22. Chang WH, Kim YH, Yoo WK, Goo KH, Park CH, Kim ST, Pascual-Leone A. rTMS with motor training modulates cortico-basal ganglia-thalamocortical circuits in stroke patients. Restor Neurol Neurosci. 2012;30(3):179-89.

23. Geyh S, Kurt T, Brockow T, Cieza A, Ewert T, Omar Z, Resch KL. Identifying the concepts contained in outcome measures of clinical trials on stroke using the international classification of functioning, disability and health as a reference. J Rehabil Med. 2004;(44 Suppl):56-62.

24. Bohannon RW, Smith MB. Interrater reliability of a modified Ashworth scale of muscle spasticity. Phys Ther. 1987;67(2):206-7.

25. Fugl-Meyer AR, Jaasko L, Leyman I, Olsson S, Steglind S. The post-stroke hemiplegic patient. 1. A method for evaluation of physical performance. Scand J Rehabil Med. 1975;7(1):13-31.
26. Yozbatiran N, Der-Yeghiaian L, Cramer SC. A standardized approach to performing the action research arm test. Neurorehabil Neural Repair. 2008;22(1):78-90.

27. Desrosiers J, Bravo G, Hebert R, Dutil E, Mercier L. Validation of the box and block test as a measure of dexterity of elderly people: reliability, validity, and norms studies. Arch Phys Med Rehabil. 1994:75(7):751-5.

28. van der Lee JH, Beckerman H, Knol DL, de Vet HC, Bouter LM. Clinimetric properties of the motor activity log for the assessment of arm use in hemiparetic patients. Stroke. 2004;35(6):1410-4.

29. Huang YZ, Lu CS, Rothwell JC, Lo CC, Chuang WL, Weng YH, Lai SC, Chen RS. Modulation of the disturbed motor network in dystonia by multisession suppression of premotor cortex. PLoS One. 2012;7(10):e47574.

30. Huang YZ, Rothwell JC, Lu CS, Wang J, Weng YH, Lai SC, Chuang WL, Hung J, Chen RS. The effect of continuous theta burst stimulation over premotor cortex on circuits in primary motor cortex and spinal cord. Clin Neurophysiol. 2009;120(4):796-801.

31. Chuang WL, Huang YZ, Lu CS, Chen RS. Reduced cortical plasticity and GABAergic modulation in essential tremor. Mov Disord. 2014; 29(4):501-7.

32. Hsu YF, Liao KK, Lee PL, Tsai YA, Yeh CL, Lai KL, Huang YZ, Lin YY, Lee $\mid H$. Intermittent theta burst stimulation over primary motor cortex enhances movement-related beta synchronisation. Clin Neurophysiol. 2011;122(11): 2260-7.

33. J C. Statistical power analysis for the behavioral sciences. Hillsdale: Lawrence Erlbaum Associates; 1988.

34. Van der Lee JH, De Groot V, Beckerman H, Wagenaar RC, Lankhorst GJ, Bouter LM. The intra- and interrater reliability of the action research arm test: a practical test of upper extremity function in patients with stroke. Arch Phys Med Rehabil. 2001;82(1):14-9.

35. van der Lee JH, Beckerman $H$, Lankhorst GJ, Bouter LM. The responsiveness of the action research arm test and the Fugl-Meyer assessment scale in chronic stroke patients. J Rehabil Med. 2001:33(3):110-3.

36. Kim H, Colantonio A. Effectiveness of rehabilitation in enhancing community integration after acute traumatic brain injury: a systematic review. Am J Occup Ther. 2010;64(5):709-19.

37. Fassett HJ, Turco CV, El-Sayes J, Lulic T, Baker S, Richardson B, Nelson AJ. Transcranial magnetic stimulation with intermittent Theta burst stimulation alters Corticospinal output in patients with chronic incomplete spinal cord injury. Front Neurol. 2017;8:380

38. Jaillard A, Martin CD, Garambois K, Lebas JF, Hommel M. Vicarious function within the human primary motor cortex? A longitudinal fMRI stroke study. Brain. 2005;128(Pt 5):1122-38.

39. Murphy $\mathrm{TH}$, Corbett D. Plasticity during stroke recovery: from synapse to behaviour. Nat Rev Neurosci. 2009;10(12):861-72.

40. George SH, Rafiei MH, Borstad A, Adeli H, Gauthier LV. Gross motor AbiLity predictS response to upper extremity rehabilitation in chronic stroke. Behav Brain Res. 2017:333:314-22.

41. Baker SN, Zaaimi B, Fisher KM, Edgley SA, Soteropoulos DS. Pathways mediating functional recovery. Prog Brain Res. 2015(218):389-412.

42. Lang CE, Schieber MH. Reduced muscle selectivity during individuated finger movements in humans after damage to the motor cortex or corticospinal tract. J Neurophysiol. 2004;91(4):1722-33.

43. Mukherjee A, Chakravarty A. Spasticity mechanisms - for the clinician. Front Neurol. 2010;1:149

44. Centonze D, Koch G, Versace V, Mori F, Rossi S, Brusa L, Grossi K, Torelli F, Prosperetti C, Cervellino A, et al. Repetitive transcranial magnetic stimulation of the motor cortex ameliorates spasticity in multiple sclerosis. Neurology. 2007:68(13):1045-50

45. Boutiere C, Rey C, Zaaraoui W, Le Troter A, Rico A, Crespy L, Achard S, Reuter F, Pariollaud F, Wirsich J, et al. Improvement of spasticity following intermittent theta burst stimulation in multiple sclerosis is associated with modulation of resting-state functional connectivity of the primary motor cortices. Mult Scler. 2017;23(6):855-63.

46. Valle AC, Dionisio K, Pitskel NB, Pascual-Leone A, Orsati F, Ferreira MJ, Boggio PS, Lima MC, Rigonatti SP, Fregni F. Low and high frequency repetitive transcranial magnetic stimulation for the treatment of spasticity. Dev Med Child Neurol. 2007;49(7):534-8.

47. Kumru H, Murillo N, Samso JV, Valls-Sole J, Edwards D, Pelayo R, ValeroCabre A, Tormos JM, Pascual-Leone A. Reduction of spasticity with repetitive transcranial magnetic stimulation in patients with spinal cord injury. Neurorehabil Neural Repair. 2010;24(5):435-41. 
48. Nardone R, Langthaler PB, Orioli A, Holler P, Holler Y, Frey VN, Brigo F, Trinka E. Effects of intermittent theta burst stimulation on spasticity after spinal cord injury. Restor Neurol Neurosci. 2017;35(3):287-94.

49. Kim DH, Shin JC, Jung S, Jung TM, Kim DY. Effects of intermittent theta burst stimulation on spasticity after stroke. Neuroreport. 2015;26(10):561-6.

50. Wupuer S, Yamamoto T, Katayama Y, Motohiko H, Sekiguchi S, Matsumura Y, Kobayashi K, Obuchi T, Fukaya C. F-wave suppression induced by suprathreshold high-frequency repetitive trascranial magnetic stimulation in poststroke patients with increased spasticity. Neuromodulation. 2013;16(3):206-11 discussion 211.

51. Stagg CJ, Wylezinska M, Matthews PM, Johansen-Berg H, Jezzard P, Rothwell $J C$, Bestmann S. Neurochemical effects of theta burst stimulation as assessed by magnetic resonance spectroscopy. J Neurophysiol. 2009;101(6):2872-7.

52. Michael N, Gosling M, Reutemann M, Kersting A, Heindel W, Arolt V, Pfleiderer B. Metabolic changes after repetitive transcranial magnetic stimulation (rTMS) of the left prefrontal cortex: a sham-controlled proton magnetic resonance spectroscopy (1H MRS) study of healthy brain. Eur J Neurosci. 2003;17(11):2462-8.

53. Strafella AP, Paus T, Fraraccio M, Dagher A. Striatal dopamine release induced by repetitive transcranial magnetic stimulation of the human motor cortex. Brain. 2003;126(Pt 12):2609-15.

54. Sala C, Piech V, Wilson NR, Passafaro M, Liu G, Sheng M. Regulation of dendritic spine morphology and synaptic function by shank and Homer. Neuron. 2001;31(1):115-30.

55. Malcolm MP, Triggs WJ, Light KE, Gonzalez Rothi LJ, Wu S, Reid K, Nadeau SE. Repetitive transcranial magnetic stimulation as an adjunct to constraintinduced therapy: an exploratory randomized controlled trial. Am J Phys Med Rehabil. 2007;86(9):707-15

56. Higgins J, Koski L, Xie H. Combining rTMS and task-oriented training in the rehabilitation of the arm after stroke: a pilot randomized controlled trial. Stroke Res Treat. 2013;2013:539146.

57. Matheson NA, Shemmell JB, De Ridder D, Reynolds JN. Understanding the effects of repetitive Transcranial magnetic stimulation on neuronal circuits. Frontiers in neural circuits. 2016;10:67.

58. Peinemann A, Reimer B, Loer C, Quartarone A, Munchau A, Conrad B, Siebner HR. Long-lasting increase in corticospinal excitability after 1800 pulses of subthreshold $5 \mathrm{~Hz}$ repetitive TMS to the primary motor cortex. Clin Neurophysiol. 2004;115(7):1519-26.

59. Weise D, Mann J, Rumpf JJ, Hallermann S, Classen J. Differential regulation of human paired associative stimulation-induced and Theta-burst stimulation-induced plasticity by L-type and T-type Ca2+ channels. Cerebral cortex. New York, NY; 1991. p. 2016.

60. Rose DK, Patten C, McGuirk TE, Lu X, Triggs WJ. Does inhibitory repetitive transcranial magnetic stimulation augment functional task practice to improve arm recovery in chronic stroke? Stroke Res Treat. 2014;2014:305236

61. Uswatte G, Taub E, Morris D, Vignolo M, McCulloch K. Reliability and validity of the upper-extremity motor activity Log-14 for measuring real-world arm use. Stroke. 2005;36(11):2493-6.

62. Taub E, Uswatte G, Mark WW, Morris DM. The learned nonuse phenomenon: implications for rehabilitation. Eura Medicophys. 2006;42(3):241-56.

\section{Ready to submit your research? Choose BMC and benefit from:}

- fast, convenient online submission

- thorough peer review by experienced researchers in your field

- rapid publication on acceptance

- support for research data, including large and complex data types

- gold Open Access which fosters wider collaboration and increased citations

- maximum visibility for your research: over $100 \mathrm{M}$ website views per year

At BMC, research is always in progress.

Learn more biomedcentral.com/submissions 